\title{
Establishment of a novel scoring model for mortality risk prediction in HIV-infected patients with cryptococcal meningitis
}

\section{Ting Zhao}

Chongqing Public Health Medical Center

\section{Xiao-Lei Xu}

Chongqing Public Health Medical Center

Jing-Min Nie

Chongqing Public Health Medical Center

\section{Xiao-Hong Chen}

Fourth Affiliated Hospital of Harbin Medical University

\section{Zhong-Sheng Jiang}

Liuzhou People's Hospital

\section{Shui-Qing Liu}

Guiyang Public Health Clinical Center

\section{Tong-Tong Yang}

Public Health Clinical Center of Chengdu

\section{Xuan Yang}

: Zhengzhou First People Hospital

\section{Feng Sun}

Chongqing Public Health Medical Center

\section{Yan-Qiu Lu}

Chongqing Public Health Medical Center

\section{Vijay Harypursat}

Chongqing Public Health Medical Center

yaokai chen ( $\square$ yaokaichen02@126.com )

Chongqing Public Health Medical Center https://orcid.org/0000-0002-9427-1367

\section{Research article}

Keywords: HIV, cryptococcal meningitis, risk factors, mortality, scoring model

Posted Date: April 1st, 2021

DOl: https://doi.org/10.21203/rs.3.rs-366320/v1 
License: (c) (i) This work is licensed under a Creative Commons Attribution 4.0 International License. Read Full License

Version of Record: A version of this preprint was published at BMC Infectious Diseases on August 10th, 2021. See the published version at https://doi.org/10.1186/s12879-021-06417-9. 


\section{Abstract}

Purpose: Cryptococcal meningitis (CM) remains a leading cause of death in HIV-infected patients, despite advances in $\mathrm{CM}$ diagnostic and therapeutic strategies. This study was performed with the aim to develop and validate a novel scoring model to predict mortality risk in HIV-infected patients with CM (HIV/CM).

Methods: Data on HIV/CM inpatients were obtained from a Multicenter Cohort study in China. Independent risk factors associated with mortality were identified based on data from 2013 to 2017, and a novel scoring model for mortality risk prediction was established. The prediction probability of the novel model was evaluated and verified using data from 2018 to 2020.

Results: We found that six predictors, including age, stiff neck, impaired consciousness, intracranial pressure, $\mathrm{CD} 4^{+} \mathrm{T}$-cell count, and urea levels, were associated with poor prognosis in HIV/CM patients. The novel scoring model could effectively identify HIV/CM patients at high risk of death on admission (area under curve $0.876 ; p \llbracket 0.001)$. When the cut-off value of 5.5 points or more was applied, the sensitivity and specificity was $74.1 \%$ and $83.8 \%$, respectively. Additionally, our scoring model showed a good discriminatory ability in the validation cohort (area under curve $0 \cdot 886 ; p \otimes 0.001$ ).

Conclusions: Our developed scoring model of six variables is simple, convenient, and accurate for screening high-risk patients with HIV/CM, which may be a useful tool for physicians to assess prognosis in HIV/CM inpatients.

\section{Introduction}

Cryptococcal meningitis (CM), caused by infection with the fungal pathogen Cryptococcus neoformans, is a leading opportunistic infection in humans, and a common cause of meningitis in adults living with Human Immunodeficiency Virus (HIV) [1-4]. Despite advances in CM diagnostic and therapeutic strategies, early mortality from CM in the developing world remains high during the therapeutic antifungal induction and consolidation phases [5]. It has been proposed that the reason for this is that early diagnosis of CM has always been clinically challenging [6-7].

Previous studies have focused on identifying key predictors of early mortality in patients who died within two to ten weeks of initiating treatment. Many potential risk factors, like age, impaired consciousness, and $\mathrm{CD} 4^{+} \mathrm{T}$-cell counts have been evaluated for their potential to predict mortality in HIV infected patients with CM (HIV/CM patients) [8-10]. Also, high intracranial pressure (ICP), blood neutrophil counts, cerebrospinal fluid (CSF) leukocyte counts, and CSF glucose levels have been evaluated in several studies as potential predictors of risk for development of $\mathrm{CM}[3,5,8]$. Although these clinical features and laboratory findings have been rigorously studied and discussed for utility as prognostication tools in HIV/CM patients, thus far, the validity of the relationship between these factors and CM mortality for this group of patients remains disappointingly unclear. Up until now, there has not been the successful development of a multi-parameter scoring system to prognostically evaluate the potential for mortality in HIV/CM patients [11]. 
We therefore comprehensively considered clinical symptoms and signs of $\mathrm{CM}$, characteristics of cerebrospinal fluid changes in $\mathrm{CM}$, and prognosis in $\mathrm{CM}$, and have developed a scoring model to accurately clinically classify and stage $\mathrm{CM}$ for attending clinicians working in primary hospital settings. Using the proposed scoring model, clinicians may accurately predict clinical outcomes in HIV/CM patients at admission from assessment of basic clinical parameters, thus facilitating expeditious and appropriate therapeutic interventions [12].

\section{Methods}

\subsection{Study design and patient population}

This retrospective multi-center cohort study included HIV-infected patients diagnosed with CM from 2013 to 2020 at the following ten Chinese medical centers: Chongqing Public Health Medical Center, The First Hospital of Changsha, Liuzhou General Hospital, Guiyang Public Health Clinical Center, Public Health Clinical Center of Chengdu, Yunnan Provincial Infectious Disease Hospital, The Fourth Affiliated Hospital of Harbin Medical University, Guangxi Longtan Hospital, The First Affiliated Hospital of Zhejiang University, and The Sixth People's Hospital of Zhengzhou. Data from all ten centers from 2013 to 2017 were used to develop the derivation cohort.

The validation cohort was based on data obtained from six of the ten mentioned medical centers above between 2018 and 2020.

\subsection{Study parameters, study endpoint, and outcomes}

The patients' clinical data, including age, headache, maximum body temperature, stiff neck, impaired consciousness, weight loss, neutrophil count, total bilirubin (TBIL), hemoglobin (HGB), platelet (PLT), urea, creatinine, CD $4^{+}$T-cell count, HIV RNA viral load, ICP, CSF glucose, clinical outcome, and other clinical data were analyzed. The observation starting point was HIV-associated CM diagnosis after admission to hospital. The observation endpoint was patients death, or at 28 days after HIV/CM diagnosis. The survival rate during the 28 days post-diagnosis was calculated as the endpoint event.

\subsection{Availability of data and materials}

The datasets generated and analysed during the current study are available on the Medical Research Platform (http://www.51yyt.org/FrontPage/login.aspx?Inviter=).

\subsection{Definitions}

Confirmation of HIV infection was established by positivity of the HIV antibody test via Western Blot, and a positive HIV RNA polymerase chain reaction [13]. The diagnostic criteria of CM were as follows [14-15]:

1. Identification of Cryptococcus in CSF via India ink staining, CSF cryptococcal antigen positivity, or CSF Cryptococcus culture positivity. 
2. CSF examination: intracranial hypertension, increased CSF protein content, reduced sugar content, mild or moderate leukocytosis in CSF.

3. Symptoms and signs: fever, headache, nausea, vomiting, altered mental state, confusion, irritability, memory impairment, behavioral changes, lethargy, stiff neck, or visual disturbances.

\subsection{Statistical analysis}

Categorical variables were compared using the Chi-squared test or Fisher's Exact test, and continuous variables were compared using Student's t-test or the Mann-Whitney U-test. The Kolmogorov-Smirnov test was used to assess the normality of the sample data distribution. Data are presented as mean \pm standard deviation, or median (interquartile range) for continuous variables, and as frequency and percentage for categorical variables. Multivariate logistical regression models were constructed using forward stepwise regression, with the primary objective of determining the demographic characteristics, clinical characteristics, and laboratory data at baseline associated with all-cause mortality (as measured at day 28). A predictive modeling strategy was used in which variables were selected for model inclusion based upon: (1) a priori knowledge from previous studies (CD $4^{+}$T-cell counts), and (2) association with outcome in the univariate analysis. Variables associated with mortality in the univariate analysis $(p<0.1)$ were included in the multivariate analysis. Variables statistically significant in the multivariate analysis $(p<0.05)$ were included in the logistic regression models. Odds ratios (OR) and $95 \%$ confidence interval $(95 \% \mathrm{Cl})$ were subsequently calculated.

The prediction score for mortality in HIV/CM patients was developed based on the final multivariate regression model. From the final logistic regression model, co-efficient scores were assigned for each factor with their respective $\beta$ coefficients. To make the scores approach an integer or relative integer, and in order to be intuitive for the user, we arbitrarily assigned the regression coefficient of ICP (0.788) as equivalent to one risk point (risk score $=1$ ), and all other $\beta$ coefficients were then divided by 0.788 to determine the score of co-efficient for each risk factor (rounded to the nearest number in units of 0.5 ). A specific risk score is derived by a calculation of each co-efficient score multiplied by each point score. The risk score for an individual was obtained by summation of the scores for each of the risk factors.

A receiver operating characteristic (ROC) curve was constructed for the scoring model. Discrimination was assessed using the area under the ROC curve, which was compared with a non-parametric approach. The ROC analysis calculated cut-off values, sensitivity, specificity, positive predictive values and negative predictive values. Finally, the Youden index was defined as (Sensitivity + Specificity) -1 [16], and the cutoff point was chosen by the best Youden index score. Statistical analyses were performed using SPSS software package for Windows (Version 23; IBM-SPSS, Inc., Chicago, IL, USA). For all tests, a $p$-value< 0.05 was considered to be statistically significant.

\subsection{Ethics statement}

This study was approved by The Ethics Committee of Chongqing Public Health Medical Center (No. 
2019-003-02-KY). All clinical investigations were conducted according to the principles expressed in the Declaration of Helsinki.

\section{Results}

A total of $903 \mathrm{HIV} / \mathrm{CM}$ patients from ten participating hospitals were screened in this study. Of these, 348 patients were excluded due to data loss (with missing test results, laboratory data, or without complete demographic characteristics), and three patients were excluded for being under 18 years of age. As a result, 386 patients were assigned to the derivation cohort, of which 328 survived and 58 died. The allcause mortality rate was $15.0 \%$. Of the 169 patients in the validation cohort, 23 patients succumbed to $\mathrm{CM}$, with an all-cause mortality rate of $13.6 \%$. A flow chart of our study population selection and study process is shown in Fig. 1.

\subsection{Comparison of clinical biochemical parameters and baseline characteristics in the derivation cohort}

To identify risk factors for mortality in $\mathrm{CM}$, we evaluated the relationship between deaths and the demographic, clinical, and laboratory characteristics of the screened individuals by performing univariate analyses on these data. The examined characteristics included demographic characteristics, clinical characteristics, and laboratory data, as summarized in Table 1. By comparing the twenty measured clinical factors between the survivors and non-survivors, six factors including impaired consciousness, stiff neck, age, TBIL, ICP, and PLT levels were found to have a significant influence on mortality risk ( $p<$ 0.0001). In addition, HIV RNA viral load, CSF glucose, and urea levels were associated significantly with patient mortality $(p<0.05)$. However, the analysis of other clinical and laboratory data, including presence of headache, weight loss, neutrophil count, creatinine, $\mathrm{CD}^{+}{ }^{+}$-cell count, sex, ART at hospital admission, and high body temperature did not differ statistically between the survival and non-survival populations $(p>0.05$, Table 1$)$. 
Table 1

Univariate analyses of baseline characteristics of HIV/CM inpatients in the derivative cohort

Variable

Variable

Survivors

Non-survivors

p-value

$(n=328)$

$(n=58)$

\section{Demographic characteristics}

Age (years, IQR)

$43(34,52)$

$263(80.2 \%)$

Male sex, n (\%)

Clinical characteristic

Maximum body temperature $\left({ }^{\circ} \mathrm{C}, \mathrm{IQR}\right)$

Stiff neck, n (\%)

Impaired consciousness, $\mathrm{n}(\%)$

Headache, $\mathrm{n}(\%)$

Weight loss, n (\%)

Laboratory data

CSF glucose (mmol/L, IQR)

Neutrophil count $\left(10^{9} / \mathrm{L}, \mathrm{IQR}\right)$

TBIL $(\mu \mathrm{mol} / \mathrm{L}, \mathrm{IQR})$

HGB (g/L, IQR)

$\operatorname{PLT}\left(\times 10^{6 / L}, \mathrm{IQR}\right)$

Urea (mmol/L, IQR)

Creatinine ( $\mu \mathrm{mol} / \mathrm{L}, \mathrm{IQR})$

White blood cell count $\left(10^{9} / \mathrm{L}, \mathrm{IQR}\right)$

CD4 ${ }^{+}$T-cell count (cells/ $\mu \mathrm{L}, \mathrm{IQR}$ )

HIV RNA ( $\log 10$ copies/mL, IQR)

ICP $\left(\mathrm{mm} \mathrm{H}_{2} \mathrm{O}, \mathrm{IQR}\right)$

ART at hospital admission, $\mathrm{n}(\%)$

* Abbreviations: CSF, cerebrospinal fluid; HGB, hemoglobin, ICP, intracranial pressure; TBIL, total bilirubin; PLT, platelet; ART, antiviral therapy; $p<0.05$ was considered statistically significant.
$37.8(36.7,39.0)$

$24(7.3 \%)$

$28(8.5 \%)$

$243(74.1 \%)$

$125(38.1 \%)$

$2.54(1.9,3.2)$

$3.4(2.3,5.4)$

$9.2(7.0,13.6)$

$115.0(98.0,130.0)$

$186(126.0,248.8)$

$3.8(3.0,5.5)$

$59.1(49.0,71.2)$

$4.6(3.4,6.5)$

$32.0(13.0,77.3)$

$5.1(3.9,5.7)$

$215.0(160.0,328.0)$

$198(51.3 \%)$

$27(46.6 \%)$

$13 \cdot 5(9.0,19.7)$

$<0.0001$

$108.0(94.3,126.0)$

0.235

$120(82,202)$

$<0.0001$

$4.9(3.7,6.8)$

0.001

$60.3(49.1,75.6)$

0.787

$4.7(3.1,7.2)$

0.939

$29.0(12.8,48.0)$

0.095

$5.4(4.9,5.9)$

0.009

$360.0(247.0,400.0)$

$<0.0001$

0.500 


\subsection{Development and internal assessment of a risk-scoring model in the derivation cohort}

The thirteen variables with a $p$-value of less than 0.1 in the univariate analysis were then entered into the multivariate logistic regression analysis. Among these variables, continuous variables were transformed into categorical variables, prior to multivariate logistic regression analysis. Table 2 shows the assignment of these variables. Multivariate analysis revealed age, stiff neck, impaired consciousness, blood urea, ICP, and CD4 + T-cell counts were potential risk factors for mortality in HIV/CM inpatients. A specific risk score (Table 3) assigned for each risk factor is derived by a calculation of each co-efficient score (Table 3) multiplied by each point score (Table 2). Based on these six potential predictors and corresponding score component value, the risk prediction model was established.

Table 2

Point scores of $386 \mathrm{HIV} / \mathrm{CM}$ inpatients in the derived cohort

\begin{tabular}{|lcccc|}
\hline & 0 & 1 & 2 & 3 \\
\hline $\mathrm{ICP}\left(\mathrm{mmH}_{2} \mathrm{O}\right)$ & $<200$ & $200-250$ & $251-350$ & $>350$ \\
\hline $\mathrm{CD} 4{ }^{+}$T-cell count $($cells $/ \mu \mathrm{L})$ & $>50$ & $\leq 50$ & & \\
\hline $\mathrm{PLT}\left(\times 10^{6} / \mathrm{L}\right)$ & $>60$ & $\leq 60$ & & \\
\hline Age $($ years $)$ & $\leq 49$ & $50-59$ & $60-69$ & \\
\hline TBIL $(\mu \mathrm{mol} / \mathrm{L})$ & $<34$ & $34-51$ & $>51$ & \\
\hline Urea $(\mathrm{mmol} / \mathrm{L})$ & $\leq 7.1$ & $>7.1$ & \\
\hline HIV RNA (log10 copies/mL) & $<5$ & $\geq 5$ & \\
\hline $\begin{array}{l}\text { *Abbreviations: ICP, intracranial pressure; PLT, platelet; TBIL, total bilirubin; } p<0.05 \text { was considered } \\
\text { statistically significant. }\end{array}$ & & & \\
\hline
\end{tabular}


Table 3

Independent predictors of mortality and corresponding score component value in HIV/CM individuals in the derived cohort

\begin{tabular}{|c|c|c|c|c|c|}
\hline Predictors & $\begin{array}{l}\text { Co- } \\
\text { efficient }\end{array}$ & $\underset{\text { value }}{p-}$ & OR (95\% Cl) & $\begin{array}{l}\text { Score of co- } \\
\text { efficient }\end{array}$ & $\begin{array}{l}\text { Score component } \\
\text { value }\end{array}$ \\
\hline Age (years) & 1.110 & $\otimes 0.001$ & $\begin{array}{l}3.034(2.050- \\
4.491)\end{array}$ & 1.5 & \\
\hline$\leq 49$ & & & & & 0 \\
\hline $50-59$ & & & & & 1.5 \\
\hline $60-69$ & & & & & 3 \\
\hline$\geq 70$ & & & & & 4.5 \\
\hline $\begin{array}{l}\text { Impaired } \\
\text { consciousness }\end{array}$ & 1.841 & $\otimes 0.001$ & $\begin{array}{l}6.304(2.740- \\
14.504)\end{array}$ & 2.5 & \\
\hline No & & & & & 0 \\
\hline Yes & & & & & 2.5 \\
\hline Stiff neck & 1.502 & 0.001 & $\begin{array}{l}4.492(1.844- \\
10.944)\end{array}$ & 2 & \\
\hline No & & & & & 0 \\
\hline Yes & & & & & 2 \\
\hline $\mathrm{ICP}\left(\mathrm{mm} \mathrm{H}_{2} \mathrm{O}\right)$ & 0.788 & $\otimes 0.001$ & $\begin{array}{l}2.200(1.604- \\
3.017)\end{array}$ & 1 & \\
\hline$<200$ & & & & & 0 \\
\hline $200-250$ & & & & & 1 \\
\hline $251-350$ & & & & & 2 \\
\hline$>350$ & & & & & 3 \\
\hline Urea (mmol/L) & 0.904 & 0.040 & $\begin{array}{l}2.470(1.041- \\
5.864)\end{array}$ & 1 & \\
\hline$\leq 7.1$ & & & & & 0 \\
\hline$>7.1$ & & & & & 1 \\
\hline $\begin{array}{l}\text { CD } 4^{+} \text {T-cell count } \\
\text { (cells } / \mu \mathrm{L})\end{array}$ & 1.505 & 0.002 & $\begin{array}{l}4.503(1.756- \\
11.547)\end{array}$ & 2 & \\
\hline$>50$ & & & & & 0 \\
\hline
\end{tabular}

*Abbreviations: ICP, intracranial pressure; PLT, platelet; TBIL, total bilirubin; OR, odds ratio; $\mathrm{Cl}$, confidence interval; $p<0.05$ was considered statistically significant. 


\begin{tabular}{|c|c|c|c|c|c|}
\hline Predictors & $\begin{array}{l}\text { Co- } \\
\text { efficient }\end{array}$ & $\begin{array}{l}p- \\
\text { value }\end{array}$ & OR $(95 \% \mathrm{Cl})$ & $\begin{array}{l}\text { Score of co- } \\
\text { efficient }\end{array}$ & $\begin{array}{l}\text { Score component } \\
\text { value }\end{array}$ \\
\hline$\leq 50$ & & & & & 2 \\
\hline
\end{tabular}

Our screening score model showed good prediction performance (reflected in the Youden index, 57.98\%), and had an area under the curve (AUC) of $0.876(95 \% \mathrm{Cl}, 0.838-0.907, \mathrm{p}<0.001)$ (Fig. 2). Using the ROC analysis, a total score greater than 5.5 was selected as the cut-off prediction value for high-risk patients. This cut-off point had a sensitivity of $74.1 \%(95 \% \mathrm{Cl}, 0.610-0.847)$ and specificity of $83.8 \%(95 \% \mathrm{Cl}$, $0.794-0.877)$ at the maximum Youden index value. With this cut-off value, the positive predictive value (PPV) and negative predictive value (NPV) were $44.8 \%(95 \% \mathrm{Cl}, 0.378-0.520)$ and $94.8 \%(95 \% \mathrm{Cl}, 0.922-$ 0.966), respectively.

To facilitate the clinical application of the predictive model, the study population with these risk factors was further divided into two subgroups (low-risk group, total score $\leq 5.5$; high-risk group, total score $>5.5$ ) according to their respective scores. The results indicated that patients with higher scores showed poorer prognosis in our cohort. In the derivation cohort, the cumulative mortality rate in the high-risk group was $44.8 \%$ (43/96), which was significantly higher than the mortality rate in the low-risk group $(15 / 290,5.2 \%)$ $(p<0.001)$ (Table 4).

Table 4

Predicted probability of mortality in HIV/CM inpatients in the derivation cohort and validation cohort.

\begin{tabular}{|llllll|}
\hline & Subgroups & Score & Survivors & Non-survivors & $\boldsymbol{p}$ \\
\hline \multirow{2}{*}{ Derivation cohort } & Low-risk group & $\leq 5.5$ & $2275(94.8 \%)$ & $15(5.2 \%)$ & $<0.001$ \\
\cline { 2 - 6 } & High-risk group & $>5.5$ & $53(55.2 \%)$ & $43(44.8 \%)$ & \\
\hline \multirow{2}{*}{ Validation cohort } & Low-risk group & $\leq 5.5$ & $1125(95.4 \%)$ & $6(4.6 \%)$ & $<0.001$ \\
\cline { 2 - 6 } & High-risk group & $>5.5$ & $21(55.3 \%)$ & $17(44.7 \%)$ & \\
\hline
\end{tabular}

\subsection{Discriminatory ability of the risk scoring model in the external validation cohort}

The performance of the new scoring model is externally verified by the validation cohort. The comparison results from the validation cohort showed that the model had good performance in predicting mortality (AUC $=0.886,95 \% \mathrm{Cl} 0.838-0.907$, Fig. 3). Comparable results were obtained in the validation datasets $(A U C=0.886,95 \% \mathrm{Cl} 0.838-0.907)$, showing good performance in predicting mortality (Fig. 3). Sensitivity of the risk scoring model was calculated to be $73.9 \%(95 \% \mathrm{Cl}, 0.516-0.898)$, specificity was $85.6 \%$ (95\% $\mathrm{Cl}, 0.789-0.909), \mathrm{PPV}$ was $44.7 \%(95 \% \mathrm{Cl}, 0.337-0.563)$, NPV was $95.4 \%(95 \% \mathrm{Cl}, 0.913-0.977)$, and the 
Youden index was $62.8 \%$. This indicates that the predictive model is effective in predicting mortality in $\mathrm{HIV} / \mathrm{CM}$ inpatients. Table 4 indicates that there was a significant difference in the cumulative mortality in the validation cohort between lower and higher predictive risk group of HIV/CM patients $(p<0.001)$ $(6 / 131,4.6 \% \& 17 / 38,44.7 \%)$.

\section{Discussion}

As is well recognized, $\mathrm{CM}$ is one of the most prevalent and potentially lethal opportunistic infectious diseases affecting HIV positive patients, and causes approximately 181,000 deaths per year worldwide [17]. All-cause mortality during the 28 days after diagnosis of CM was $15.0 \%$ in our study, which is concernedly high, although lower than that observed in other studies [18-20]. It is therefore vital for clinicians to determine the risk factors that favor the development of HIV/CM. Although many past studies have investigated potential prognostic factors for $\mathrm{CM}$, consensus regarding prognostic risk factors remains elusive due to utilization of different therapeutic regimens in different studies or different regions.

In the present study, we developed and validated a risk scoring model for HIV/CM using the data of 555 subjects afflicted with HIV-associated CM. After verifying the scoring model, the following six risk predictors were selected: age, stiff neck, impaired consciousness, blood urea, ICP, and CD4 + T-cell counts of HIV/CM patients at admission. Based on these risk factors, a novel model for HIV/CM prognostication was built, which, to the best of our knowledge, is the first prognostic assessment model for use in patients with HIV/CM.

Compared to several previous studies which have also identified some similar risk factors associated with poor prognosis in CM patients [9, 21-25], the present study differs in the following aspects: 1) All of our study participants were HIV-infected persons while the study participants in the majority of previous studies were HIV-negative persons, and the total number of participants in this study was 555 , which is a relatively large sample size with regards to these types of studies. 2) Some studies chose neurocognitive impairment, seizures, neurological sequelae, relapse, or treatment effectiveness as outcome indicators in their analysis of risk factors for HIV/CM, rather than death as an outcome. 3) Our study established a convenient and novel predictive model based on the previously mentioned risk factors, and the model was then further validated using data from another $169 \mathrm{HIV} / \mathrm{CM}$ patients.

In our study, mortality was higher for patients older than 50 years of age, and risk scores increased by as much as 1.5 points for each additional 10 years of age. A recent study found that 8-week mortality for patients who were $\geq 50$ years was higher than those who were < 50 years (28.6\% vs $17.3 \%)$ [26]. In a retrospective analysis, older age (> 50 years) was independently associated with 2-week mortality of patients with HIV complicated with $\mathrm{CM}$ [8]. Moreover, patients aged $\geq 50$ years were almost 4 times more likely to die than those aged < 50 years [8]. Qu et al. reported that the survival rate of patients with CM aged over 60 years was worse than that of those younger than 60 years of age [27]. These findings indicate that advanced age ( $\geq 50$ years old) is an important predictor of mortality risk in patients with 
CM. Globally, the life span of people living with HIV is now much longer than in the past, suggesting that clinicians need to be focus on the age of patients, pay appropriate attention to the patient's clinical condition, and make appropriate therapeutic adjustments timeously.

Elevated ICP is common during the diagnostic workup for CM, with more than half of HIV/CM patients reportedly having an opening ICP of over $250 \mathrm{mmH}_{2} \mathrm{O}$, and more than a quarter having an opening ICP of above $350 \mathrm{mmH}_{2} \mathrm{O}$ [28]. Raised ICP at baseline diagnosis of $\mathrm{CM}\left(\geq 250 \mathrm{mmH}_{2} \mathrm{O}\right)$ is associated with altered mental status, visual and hearing loss, other symptoms of cranial nerve dysfunction, and even impaired consciousness or death. In a large trial of CM treatment, almost all the early deaths (13/14) and

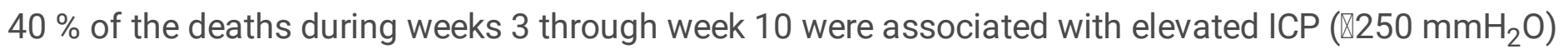
[29]. Graybill and colleagues also found that short-term survival of HIV/CM patients was significantly lower in the 250-349 $\mathrm{mmH}_{2} \mathrm{O}$ pressure group and $>350 \mathrm{mmH}_{2} \mathrm{O}$ pressure group, than those with pretreatment opening pressures $₫ 250 \mathrm{mmH}_{2} \mathrm{O}$ (according to Kaplan-Meier analysis) [30]. Another observational retrospective study also found that HIV/CM patients in the high ICP group $\left(>250 \mathrm{mmH}_{2} \mathrm{O}\right)$ had a higher mortality rate $(21 / 57,36.8 \%)$ than those who had a normal ICP at baseline $(6 / 23,26.1 \%)$ [31]. Consistent with the findings of these earlier trials, in our study, elevated ICP did negatively impact on outcomes at 4 weeks. Stepwise multivariate analysis indicated that as ICP increased, the risk of death for $\mathrm{HIV}$-infected patients with $\mathrm{CM}$ also increased. Aggressive management of increased ICP is therefore suggested in the treatment of CM, with the goal of achieving an ICP of $200 \mathrm{H}_{2} \mathrm{O}$ or less, or $50 \%$ of the initial opening pressure measured [32]. At the same time, current guidelines call for subsequent appropriate drug treatment of CM to maintain normal ICP to control symptoms of intracranial hypertension [33]. Of note, CSF opening pressures should be measured as an integral part of the initial evaluation. If the initial ICP is normal, the ICP should be further measured at least once more. For patients with elevated baseline ICP (> $250 \mathrm{mmH}_{2} \mathrm{O}$ ), additional LP (including daily LP) may be performed as necessary until the pressure is adequately controlled [34]. In a study of 26 patients with CM, 7 of 14 patients who did not have their initial ICP measured developed neuropathies during therapy, compared to only 1 of 12 patients who underwent ICP monitoring [35]. This suggests that deviations from standard ICP management could likely lead to poor neurological outcomes. As such, these analyses support the call for continued vigilance regarding ICP management during the window of opportunity shortly after diagnosis of HIV/CM, including timely monitoring of CSF pressure and effective control of ICP during antifungal therapy.

Low CD $4+$ T-cell count is associated with a poor prognosis of CM. Pastick's study found that a CD $4+T$ cell count of $<50$ cells/ $\mu \mathrm{L}$ was an important predictor of epilepsy in patients with HIV/CM [9]. A study in Uganda indicated that a CD4 + T-cell count of $<50$ cells $/ \mu \mathrm{L}$ was associated with higher 18-week mortality, compared to a CD4 + T-cell count of 50-100 cells/ $\mu \mathrm{L}$ and 100-200 cells/ $\mu \mathrm{L}$ [36]. This finding was similar to our observation in the present study, suggesting that monitoring of CD $4+$ T-cell levels in HIV/CM helps identify patients with increased risk of poor prognosis. Impaired consciousness and stiff neck are common clinical manifestations and symptoms in patients with $\mathrm{CM}$, and their incidence was significantly higher in patients with unfavorable outcomes [25]. Wu et al. reported that the presence of impaired 
consciousness increased the risk of poor prognosis by a factor of 4.4 [24]. High urea nitrogen level is often a manifestation of abnormal renal function. Our results in this study found that high urea nitrogen was an important risk factor for mortality in HIV/CM patients, which indicates that periodic screening for, and timely normalization of urea nitrogen were necessary in patients with $\mathrm{CM}$.

In summary, our novel scoring model is likely to be useful for predicting outcomes in patients with $\mathrm{HIV/CM}$. Utilizing our scoring model, clinicians may stratify affected patients so that they may decide upon appropriate patient-specific treatment regimens, or, if indicated, make early referrals to specialist care, particularly in primary care settings. Higher scores obtained via our novel system may serve as an early warning for this particular group of patients, as this should alert attending clinicians that enhanced or more efficacious antifungal regimens may be indicated in particular HIV/CM patients. Low scores may indicate that affected patients may be placed in a milder risk category, which may help reduce inappropriate utilization and wastage of expensive medical resources [37]. Additionally, the listed predictive risk factors in our study would be paid additional attention to by attending physicians along the entire course of the patient's illness, as positive changes to the risk factors during the illness course, may positively influence HIV/CM outcomes. It is hoped that our novel scoring model may be incorporated into the diagnostic and therapeutic workup for newly-diagnosed HIV/CM patients so as to improve outcomes in this patient population.

The present study has limitations. First, as our study was retrospective in nature, it therefore may be susceptible to the various limitations inherent to retrospective studies in general. Second, the participants in our study were all Chinese nationals, and whether our proposed scoring model can successfully be used for other population or racial groups is unknown, and warrants further research. Additionally, there are a few potential risk factors for which we did not have adequate information in our data set, and were not included in our data analysis, and inclusion of these risk factors into our model may further improve the prognostic accuracy of our mortality risk score. These potential risk factors include cryptococcal antigen titers (CrAg titers) in both serum and CSF, body mass index (BMI), CSF protein levels, CSF chloride levels, dose and course length for Amphotericin B, and other central nervous system symptoms (e.g., vision loss, hearing loss, etc.). There is evidence that the higher CrAg titers in both serum and CSF are associated with an increased risk of mortality in HIV/CM [38], and thus its inclusion as a potential predictive risk factor in the development of future risk scores for HIV/CM patients should be considered. However, hospitals and clinics that do not have the capacity to perform these additional tests may safely use our novel scoring model in order to accurately stratify risk in HIV patients with CM.

\section{Conclusions}

In conclusion, our study outlines a simple and useful scoring model developed to predict mortality in $\mathrm{HIV/CM}$. The sensitivity, specificity, and accuracy of the scoring system after verification are meaningful and applicable to clinical medical practice in areas where HIV/CM prevalence and mortality remains notably high. We envision that our scoring model may be widely used in these settings in order to effect demonstrable reductions in HIV/CM mortality. 


\section{Abbreviations}

CM (cryptococcal meningitis), PLT (platelet), ROC (receiver operating characteristic), TBIL (total bilirubin), HGB (hemoglobin), AmB, amphotericin B; ART, antiviral therapy; OR (odds ratios), 95\% Cl (95\% confidence interval), HIV (Human Immunodeficiency Virus), WHO (World Health Organization), SPSS (Statistical Package for the Social Sciences).

\section{Declarations}

\section{Ethics approval and consent to participate}

This study was endorsed by the Ethics Committee of Chongqing Public Health Medical Center (registry number 2019-003-02-KY). All clinical investigations were conducted according to the principles expressed in the Declaration of Helsinki.

\section{Funding}

This work was supported by the National Science and Technology Major Project of China During the 13th Five-year Plan Period (2018ZX10302104); the Joint Medical Research Project of Chongqing Municipal Science and Technology Bureau and Chongqing Municipal Health Commission (2019ZDXM012); Youth Scientific Research and Innovation Fund Project of Chongqing Public Health Medical Center (2019QNKYXM05), and the Capacity Improvement Plan Project of Units appointed by the Chongqing Municipal Health Commission (2019NLTS003).

\section{Author Contributions}

Yaokai Chen and Jinmin Nie: Study design. Tongtong Yang, Shuiqing Liu, Zhongsheng Jiang, Xiaohong Chen, Xuan Yang, Feng Sun, Yanqiu Lu and Yanqiu Lu: Data acquisition. Ting Zhao and Xiao Lei Xu: Statistical analyses and drafting of manuscript. The manuscript was reviewed, revised, and proofread by Vijay Harypursat and Yaokai Chen.

\section{Declaration of competing interest}

The authors declare that they have no competing interests.

\section{Acknowledgement}

We wish to express our gratitude to the institutions that played critical roles in the data collection process. We also would like to acknowledge staff at each participating site who have assisted in obtaining ethics and operational approval for the study.

\section{References}


[1] Loyse A, Wilson D, Meintjes G, et al. Comparison of the early fungicidal activity of high-dose fluconazole, voriconazole, and flucytosine as second-line drugs given in combination with amphotericin B for the treatment of HIV-associated cryptococcal meningitis. Clin Infect Dis 2012;54:121-128. DOI: $10.1093 / \mathrm{cid} / \mathrm{cir} 745$.

[2] Li Z, Liu Y, Chong Y, et al. Fluconazole plus flucytosine is a good alternative therapy for non-HIV and non-transplant-associated cryptococcal meningitis: A retrospective cohort study. Mycoses. 2019;62. DOI: $10.1111 /$ myc. 12944.

[3] Ssebambulidde K, Bangdiwala AS, Kwizera R, et al. Symptomatic Cryptococcal Antigenemia Presenting as Early Cryptococcal Meningitis With Negative Cerebral Spinal Fluid Analysis. Clin Infect Dis 2019; 68:2094-2098. DOI: 10.1093/cid/ciy817.

[4] Wu X, Shen Y. Management of human immunodeficiency virus-associated cryptococcal meningitis: Current status and future directions. Mycoses.2019;62. DOI: 10.1111/myc.12977.

[5] Musubire AK, Meya DB, Rhein J, et al. Blood neutrophil counts in HIV-infected patients with cryptococcal meningitis: Association with mortality. PLoS One 2018;13: e0209337. DOI: 10.1371/journal.pone.0209337.

[6] Kashef Hamadani BH, Franco-Paredes C, Mccollister B. Cryptococcosis and cryptococcal meningitis: New predictors and clinical outcomes at a United States academic medical centre. Mycoses 2018;61:314320. DOI: $10.1111 /$ myc. 12742 .

[7] Abd El-Wahab EW, Farrag T, and Metwally M. A clinical rule for the prediction of meningitis in HIV patients in the era of combination antiretroviral therapy. Trans R Soc Trop Med Hyg 2020;114: 264-275. DOI: 10.1093/trstmh/trz107.

[8] Jarvis JN, Bicanic T, Loyse A, et al. Determinants of mortality in a combined cohort of 501 patients with HIV-associated Cryptococcal meningitis: implications for improving outcomes. Clin Infect Dis 2014;58:736-745. DOI: 10.1093/cid/cit794.

[9] Pastick KA, Bangdiwala AS, Abassi M, et al. Seizures in Human Immunodeficiency Virus-Associated Cryptococcal Meningitis: Predictors and Outcomes. Open Forum Infect Dis 2019;6:ofz478.

DOI: 10.1093/ofid/ofz478.

[10] Tsai WC, Lien CY, Lee JJ, et al. The clinical characteristics of adult cryptococcal meningitis patients who died within one year of treatment with a focus on those with early mortality. J Clin Neurosci 2019;67:80-84. DOI: 10.1016/j.jocn.2019.06.015.

[11] Tenforde MW, Gertz AM, Lawrence DS, et al. Mortality from HIV-associated meningitis in sub-Saharan Africa: a systematic review and meta-analysis. J Int AIDS Soc 2020;23: e25416. DOI: 10.1002/jia2.25416. 
[12] Lofgren S, Abassi M, Rhein J, et al. Recent advances in AIDS-related cryptococcal meningitis treatment with an emphasis on resource limited settings. Expert Rev Anti Infect Ther 2017;15: 331-340. DOI: 10.1080/14787210.2017.1285697.

[13]World Health Organization. Consolidated Guidelines on HIV Testing Services: 5Cs: Consent, Confidentiality, Counselling, Correct Results and Connection 2015. https://doi.org/10.13140/RG.2.1.22 63.6882; 2015, Accessed date: 14 April 2020.

[14] Xu L, Tao R, Wu J, et al. Short-Course Rather Than Low-Dose Amphotericin B May Exert Potential Influence on Mortality in Cryptococcal Meningitis Patients Treated With Amphotericin B Plus Flucytosine Alone or in Combination With Fluconazole. Front Microbiol 2019;10: 2082.

DOI: $10.3389 /$ fmicb.2019.02082.

[15] Rajasingham R, Wake RM, Beyene T, et al. Cryptococcal Meningitis Diagnostics and Screening in the Era of Point-of-Care Laboratory Testing. Journal of Clinical Microbiology 2019; 57:e01238-18. DOI: 10.1128/JCM.01238-18.

[16] Luo M, Yang Y, Xu J, et al. A new scoring model for the prediction of mortality in patients with acute kidney injury. Sci Rep 2017;7:7862. DOI: 10.1038/s41598-017-08440-w.

[17] Williamson PR. The relentless march of cryptococcal meningitis. The Lancet Infectious Diseases 2017;17:790-791. DOI: 10.1016/S1473-3099(17)30245-1.

[18] Lessells R J, Mutevedzi P C, Heller T, et al. Poor long-term outcomes for cryptococcal meningitis in rural South Africa. S Afr Med J 2011;101:251-252. DOI: 10.7196/samj.4378.

[19] Kendi C, Penner J, Koech J, et al. Predictors of outcome in routine care for Cryptococcal meningitis in Western Kenya: lessons for HIV outpatient care in resource-limited settings. Postgraduate Medical Journal 2013;89:73-77. DOI: 10.1136/postgradmedj-2012-130823.

[20] Estelle P, John K, and Pierre DB. Long term mortality and disability in Cryptococcal Meningitis: a systematic literature review. Clin Infect Dis 2017;66: 1122-1132. DOI: 10.1093/cid/cix870.

[21] Anekthananon T, Manosuthi W, Chetchotisakd P, et al. Predictors of poor clinical outcome of cryptococcal meningitis in HIV-infected patients. Int J STD AIDS 2011;22:665-670. DOI:

10.1258/ijsa.2011.010538.

[22] Carlson RD, Rolfes MA, Birkenkamp KE, et al. Predictors of neurocognitive outcomes on antiretroviral therapy after cryptococcal meningitis: a prospective cohort study. Metab Brain Dis 2014;29: 269-279. DOI: 10.1007/s11011-013-9476-1.

[23] Xu L, Zhang X, Guo Y, et al. Unique clinical features of cryptococcal meningitis among Chinese patients without predisposing diseases against patients with predisposing diseases. Med Mycol 2019;57:944-953. DOI: 10.1093/mmy/myy154. 
[24] Wu L, Xiao J, Song Y, Gao G, Zhao H. The clinical characteristics and outcome of cryptococcal meningitis with AIDS in a tertiary hospital in China: an observational cohort study. BMC Infect Dis. 2020 Dec 1;20(1):912. DOI: 10.1186/s12879-020-05661-9.

[25] Hakyemez IN, Erdem H, Beraud G, Lurdes M, et al. Prediction of unfavorable outcomes in cryptococcal meningitis: results of the multicenter Infectious Diseases International Research Initiative (ID-IRI) cryptococcal meningitis study. Eur J Clin Microbiol Infect Dis. 2018 Jul;37(7):1231-1240. DOI: 10.1007/s10096-017-3142-1.

[26] Song W, Shen YZ, Wang ZY, et al. Clinical features and treatment outcomes of human immunodeficiency virus-associated cryptococcal meningitis: a 2-year retrospective analysis. Chin Med J (Engl). 2020 Dec 5;133(23):2787-2795. DOI: 10.1097/CM9.0000000000001191.

[27] Qu J, Zhou T, Zhong C, Deng R, Lü X. Comparison of clinical features and prognostic factors in HIVnegative adults with cryptococcal meningitis and tuberculous meningitis: a retrospective study. BMC Infect Dis. 2017 Jan 10;17(1):51. doi: 10.1186/s12879-016-2126-6. DOI: 10.1186/s12879-016-2126-6.

[28] Chang CC, Perfect JR. Repeated therapeutic lumbar punctures in cryptococcal meningitis - necessity and/or opportunity? Curr Opin Infect Dis. 2016 Dec;29(6):539-545. DOI:

10.1097/QCO.0000000000000315.

[29] van der Horst CM, Saag MS, Cloud GA, Hamill RJ, Graybill JR, Sobel JD, Johnson PC, Tuazon CU, Kerkering T, Moskovitz BL, Powderly WG, Dismukes WE. Treatment of cryptococcal meningitis associated with the acquired immunodeficiency syndrome. National Institute of Allergy and Infectious Diseases Mycoses Study Group and AIDS Clinical Trials Group. N Engl J Med. 1997 Jul 3;337(1):15-21. DOI: 10.1056/NEJM199707033370103.

[30] Graybill JR, Sobel J, Saag M, et al. Diagnosis and management of increased intracranial pressure in patients with AIDS and cryptococcal meningitis. The NIAID Mycoses Study Group and AIDS Cooperative Treatment Groups. Clin Infect Dis. 2000 Jan;30(1):47-54. DOI: 10.1086/313603.

[31] de Vedia L, Arechavala A, Calderón MI, et al. Relevance of intracranial hypertension control in the management of Cryptococcus neoformans meningitis related to AIDS. Infection. 2013 Dec;41(6):1073-7. DOI: $10.1007 / \mathrm{s} 15010-013-0538-4$.

[32] Srichatrapimuk S, Sungkanuparph S. Integrated therapy for HIV and cryptococcosis. AIDS Res Ther. 2016 Nov 29;13(1):42. DOI: 10.1186/s12981-016-0126-7.

[33] Perfect JR, Dismukes WE, Dromer F, et al. Clinical practice guidelines for the management of cryptococcal disease: 2010 update by the infectious diseases society of america. Clin Infect Dis. 2010 Feb 1;50(3):291-322. DOI: 10.1086/649858. 
[34] Pappas PG. Managing cryptococcal meningitis is about handling the pressure. Clin Infect Dis. 2005 Feb 1;40(3):480-2. doi: 10.1086/427222. Epub 2005 Jan 10. DOI: 10.1086/427222.

[35] Shoham S, Cover C, Donegan N, et al. Cryptococcus neoformans meningitis at 2 hospitals in Washington, D.C.: adherence of health care providers to published practice guidelines for the management of cryptococcal disease. Clin Infect Dis. 2005 Feb 1;40(3):477-9. DOI: 10.1086/427213.

[36] Tugume L, Rhein J, Hullsiek KH, et al. HIV-Associated Cryptococcal Meningitis Occurring at Relatively Higher CD4 Counts. J Infect Dis. 2019 Feb 23;219(6):877-883. doi: 10.1093/infdis/jiy602. DOI: 10.1093/infdis/jiy602.

[37] Loyse A, Burry J, Cohn J, et al. Leave no one behind: response to new evidence and guidelines for the management of cryptococcal meningitis in low-income and middle-income countries. The Lancet Infectious Diseases 2019;19:e143-e147. DOI: 10.1016/S1473-3099(18)30493-6.

[38] Wake RM, Britz E, Sriruttan C, et al. High Cryptococcal Antigen Titers in Blood Are Predictive of Subclinical Cryptococcal Meningitis Among Human Immunodeficiency Virus-Infected Patients. Clin Infect Dis 2018;66:686-692. DOI: 10.1093/cid/cix872.

\section{Figures}




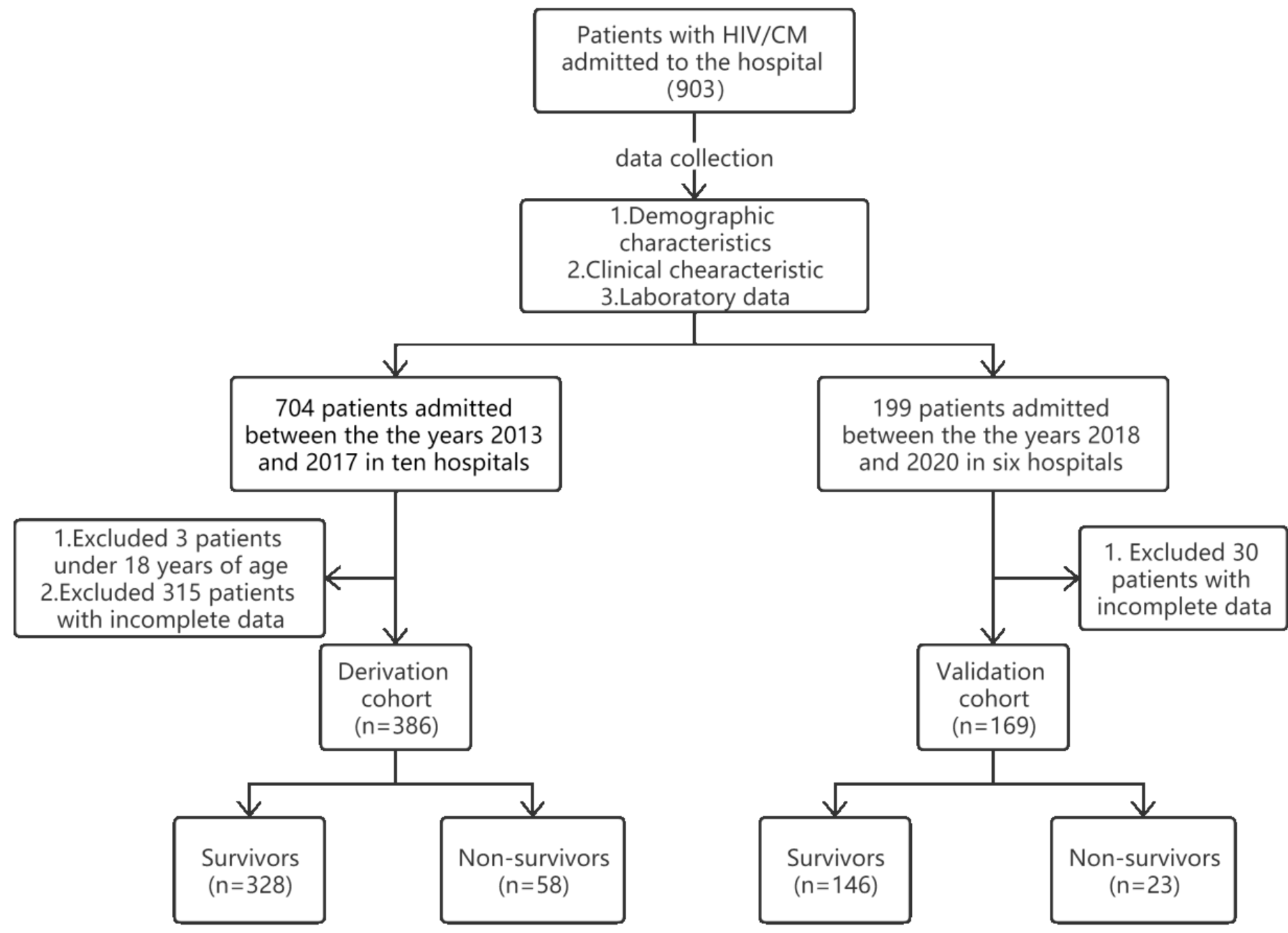

Figure 1

Flow chart of inclusion and exclusion of study participants

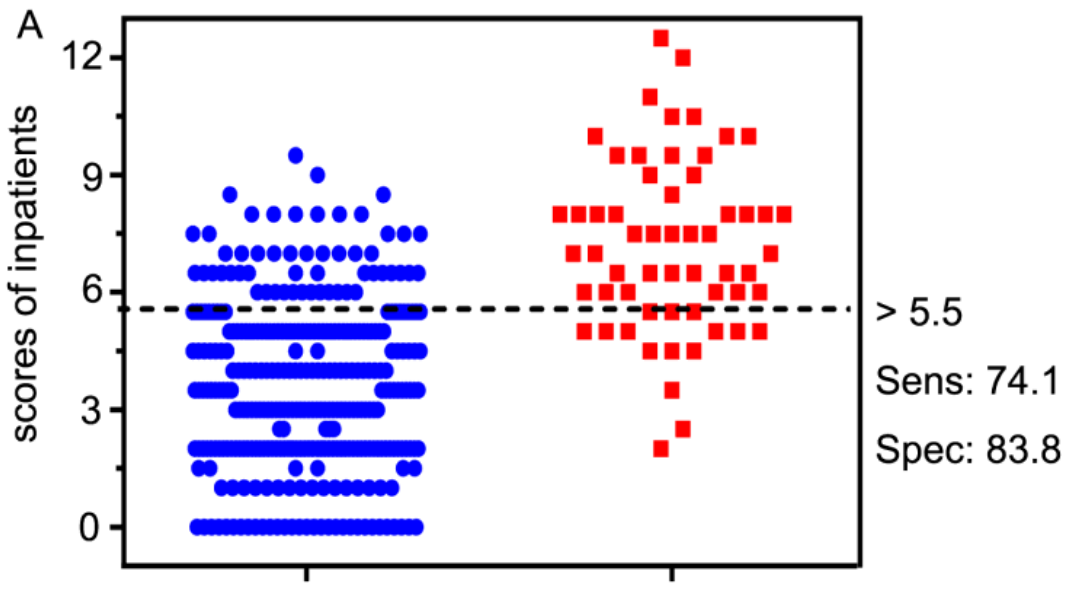

Survivors

Non-survivors

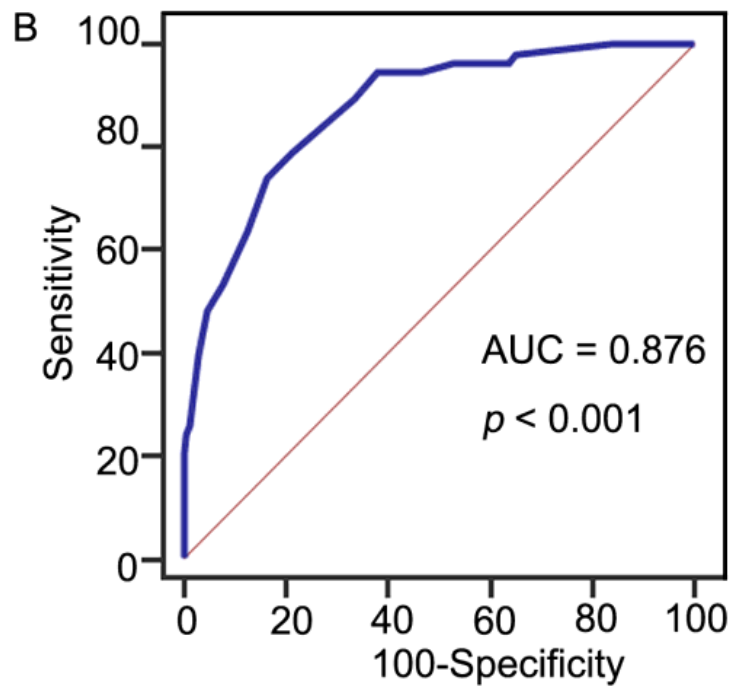


Figure 2

Mortality risk prediction rules for $386 \mathrm{HIV} / \mathrm{CM}$ inpatients in the derivation cohort. (A) Interactive dot diagram. The total score calculated by the prediction model labels the vertical axis of the above plot, and the clinical outcome of a patient during hospitalization marks the horizontal axis. The scores for each patient are displayed as dots on two vertical axes. The horizontal line indicates the cut-off point with the best separation. When using a score of 5.5 as the cut-off value to predict the mortality risk of HIV/CM inpatients, the sensitivity of our scoring system was $74.1 \%(95 \% \mathrm{Cl}, 0.610-0.847)$, its specificity was $83.8 \%$ (95\% Cl,0.794-0.877). (B) The ROC curve. Each point on the ROC curve represents a sensitivity/specificity pair corresponding to a particular decision threshold. The AUC is a measure of how well a parameter can distinguish between two groups (survivors/non- survivors). ROC, Receiver operator characteristic; AUC, area under the ROC curve; Sens, sensitivity; Spec, specificity.
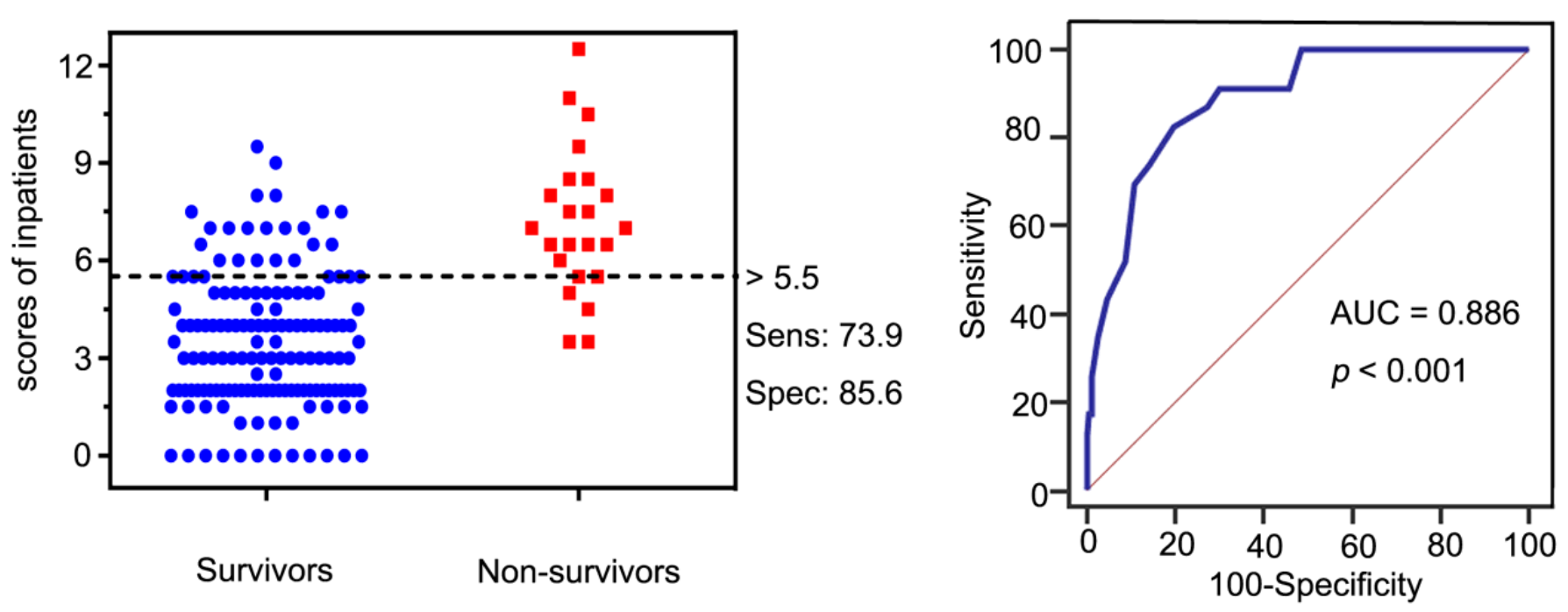

\section{Figure 3}

Mortality risk prediction rules for $169 \mathrm{HIV} / \mathrm{CM}$ inpatients in validation cohort. (A) Interactive dot diagram. The total score calculated by the prediction model labels the vertical axis of the above plot, and the clinical outcome of a patient during hospitalization marks the horizontal axis. The scores for each patient are displayed as dots on two vertical axes. The horizontal line indicates the cut-off point with the best separation. When using a score of 5.5 as the cut-off value to predict the mortality risk of HIV/CM inpatients, the sensitivity of the risk scoring system was calculated to be $73.9 \%$ ( $95 \% \mathrm{Cl}, 0.516-0.898)$, its specificity was $85.6 \%(95 \% \mathrm{Cl}, 0.789-0.909)$. (B) The ROC curve. Each point on the ROC curve represents a sensitivity/specificity pair corresponding to a particular decision threshold. The AUC is a measure of how well a parameter can distinguish between two groups (survivors/non- survivors). ROC, Receiver operator characteristic; AUC, area under the ROC curve; Sens, sensitivity; Spec, specificity. 